\title{
Cardionephrology: Proposal for a Futuristic Educational Approach to a Contemporary Need
}

\author{
Amir Kazory $^{a}$ Peter A. McCullough ${ }^{\text {b-d }}$ Janani Rangaswami ${ }^{e, f}$ \\ Claudio Ronco ${ }^{\mathrm{g}} \mathrm{h}$ \\ aDivision of Nephrology, Hypertension, and Renal Transplantation, University of Florida, \\ Gainesville, FL, USA; ${ }^{b}$ Baylor University Medical Center, Dallas, TX, USA; ${ }^{c}$ Baylor University \\ Medical Center, Baylor Heart and Vascular Institute, Dallas, TX, USA; ${ }^{\mathrm{d}}$ Baylor Jack and Jane \\ Hamilton Heart and Vascular Hospital, Dallas, TX, USA; ${ }^{e}$ Division of Nephrology, Einstein \\ Medical Center, Philadelphia, PA, USA; ${ }^{f}$ Sidney Kimmel Medical College of Thomas Jefferson \\ University, Philadelphia, PA, USA; ${ }^{9}$ Department of Nephrology, San Bortolo Hospital,

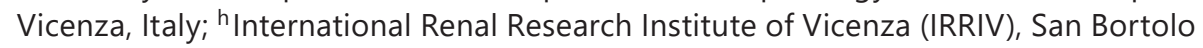 \\ Hospital, Vicenza, Italy
}

\section{Keywords}

Cardionephrology · Curriculum · Education · Workgroup

\begin{abstract}
The field of cardiorenal medicine is vast, rapidly expanding, and complex. Conventional nephrology training programs provide the fellows with the necessary core knowledge to provide general care for patients with renal and cardiovascular diseases. However, there is a need for focused training of interested physicians to master the specialized aspects of these exceedingly common clinical scenarios and optimize the care of such patients. A cardionephrologyfocused training can add value to the nephrology subspecialty and potentially increase its attractiveness for a significant subset of trainees. Herein, we provide a proposal for the framework and content of such an educational activity. Creation of an international multidisciplinary workgroup to formulate a comprehensive curriculum for a dedicated cardionephrology track would be the first step. A variety of practical aspects such as implementation methods, the identification of the required skills, and the development of educational assessment tools are discussed. While this proposal primarily focuses on the integration of the curriculum into the training of nephrology fellows, it would also be appropriate (albeit in a modified and customized format) for a wider range of trainees, including cardiology fellows.
\end{abstract}




\section{Background}

With the increase of the aging population as well as the substantial improvement in the outcomes of ischemic events, the prevalence of patients with cardiovascular diseases such as heart failure (HF) is on the rise [1,2]. We observe constant growth in the use of mechanical circulatory support devices, the emergence of novel medical therapies for HF, and the widespread application of coronary interventions. These advancements not only have led to a better understanding of the reciprocal mechanisms linking the heart and kidneys in various clinical settings $[3,4]$, but have also generated a need for experienced nephrologists with a deep understanding of the renal aspects of these therapies.

On the other hand, in recent years, there have been several exciting developments in the field of nephrology with potentially significant implications for the care of patients suffering from cardiac diseases. These advancements range from the development of robust serum and urinary biomarkers to enable early risk stratification and the detection of tubular injury, to a better understanding of the pathophysiologic mechanisms underlying renal dysfunction in sepsis, and the emergence of renal replacement therapies with highly advanced technologies. Besides, a number of new adjunct tools have been added to the armamentarium of both nephrologists and cardiologists for more precise assessment of volume status, such as pointof-care ultrasound scans, bioimpedance technology, and devices integrated within dialysis machines that can perform live monitoring of changes in intravascular volume. As such, the clinical and technological interface between nephrology and cardiology is rapidly expanding [5].

\section{The Proposal}

While clinical scenarios have opened new arenas for a multidisciplinary approach to complex cardiorenal diseases, their constantly expanding spectrum mandates the training of new generations of physicians to focus on this specific population. The kidney-heart crosstalk is known to be continuous, and is further amplified and complicated by the ever-changing clinical profiles of patients [6]. Current general nephrology training, while helpful for providing the core knowledge and skills needed for managing patients with a broad spectrum of renal diseases, does not seem to be sufficient for covering the rapidly evolving field of cardiorenal medicine.

Previously, a proposal was made for an innovative program to enhance the education of both cardiology and nephrology training [7]. As knowledge and confidence in cardiorenal medicine are critical for the physicians providing care for these complex patients, it is important to develop comprehensive, practical, and widely accepted cardionephrology curricula and incorporate these into existing nephrology training programs. Herein, we provide more details on the framework and content of such an educational activity which are, in our opinion, the core knowledge and skills needed to provide optimal care for these patients. While this proposal primarily focuses on the integration of the curriculum into the training of nephrology fellows, it would also be appropriate, albeit in a modified and customized format, for a wider range of trainees including cardiology fellows and heart failure fellows in so-called advanced "super fellowships" as well as interested internal medicine residents.

Barriers to providing any new educational program usually include a lack of external funding and faculty time constraints. The objective of this proposal is therefore to develop a cardionephrology curriculum without external funding that requires a minimal time commitment from faculty members due to its multidisciplinary nature and use of already 
existing resources. This novel curriculum is meant to shape the foundation for a more comprehensive view of cardiorenal medicine than the conventional nephrology fellowship training. It could also pave the way for an advanced fellowship program in the future.

We also propose that a workgroup, comprising nephrologists (including those focused on critical care nephrology), cardiologists with a variety of interests and expertise (e.g., general, electrophysiology, and interventional) as well as intensivists with expertise in cardiovascular or renal medicine, gathers to formally design the blueprint of a cardionephrology curriculum, oversee its implementation, and assess its outcomes. The Acute Dialysis Quality Initiative (ADQI) workgroup experience could be used as a successful platform for such an approach [5]. The workgroup can also decide on the development or collection of the pertinent reading material and educational resources for this purpose. The following can be a starting point.

\section{Curriculum Framework}

The curriculum would be implemented over a number of months that are preferably consecutive. Depending on the scope of practice of the teaching facility, it can be offered as a cumulative block of elective time of the nephrology training, preferably during the second year of fellowship. Alternatively, it can be offered as a "specialty track" such as those currently offered by a number of centers (e.g., clinician-educator, advanced glomerular disease, and dialytic therapies). In countries like France where the specialty training (i.e., the Diplôme d'Études Spécialisées, DES) is typically offered in distinct 6-month blocks, a full semester could be spent in the cardiology service to accomplish this goal.

The direct, hands-on clinical experience will be the predominant aspect of the curriculum to promote self-efficacy in real-life clinical cardiorenal medicine. To enhance academic skills and independent learning, the trainees will be required to develop a quality improvement or clinical research project that focuses on a particular topic in cardiorenal medicine. They will also be asked to provide at least 1 presentation for the common nephrology-cardiology grand rounds, as well as 1 journal club presentation per month moderated by a faculty member, with 2 half days a week designated for independent study and the completion of the project. Moreover, appropriate reading material and Web-based educational resources will be provided by the attending physicians throughout this elective.

\section{Curriculum Content}

A variety of topics related to cardiorenal medicine can be considered for inclusion in the curriculum, relating to both acute and chronic processes as well as the inpatient or outpatient setting (Table 1). While the core knowledge would remain the same to ensure consistency, the interest of the trainee as well as the expertise and clinical focus area of the attending physicians would supplement and guide the specific subject materials covered. Obviously, the patient population as well as any related local disciplines and programs (e.g., the presence of an active cardiothoracic surgery service or an extracorporeal membrane oxygenation [ECMO] program) will affect the educational content and value of the cardionephrology curriculum.

Familiarity with the relevant consensus guidelines of professional societies (e.g., the HF guidelines of the American Heart Association) should be part of this educational program. This could be incorporated in the form of didactic review sessions focused on specific topics (e.g., blood pressure goals and management in HF). The basic foundations of cardiorenal medicine including proposed underlying mechanisms as well as various existing classification schema and evolving concepts (e.g., the dynamic role of congestion and renal dysfunction 
Kazory et al.: Cardionephrology: A Futuristic Educational Approach to a Contemporary Need

Table 1. Example of proposed topics for the cardionephrology curriculum

\begin{tabular}{|c|c|}
\hline Topic & Details \\
\hline \multicolumn{2}{|l|}{ Inpatients } \\
\hline Volume status assessment & Use of bioimpedance analysis, lung and IVC ultrasound \\
\hline Fluid overload therapy & $\begin{array}{l}\text { Use of SCUF, urgent-start PD, optimization of PD modalities and regimens for } \\
\text { sodium and water removal if ESRD, and evidence-based use of diuretics in } \\
\text { landmark studies (e.g., DOSE and CARRESS-HF) }\end{array}$ \\
\hline $\begin{array}{l}\text { AKI (ADHF and postcardiac } \\
\text { surgery) }\end{array}$ & $\begin{array}{l}\text { Use of biomarkers for early diagnosis and prognostication, and impact of } \\
\text { changes in renal function (WRF/IRF) on outcomes }\end{array}$ \\
\hline $\begin{array}{l}\text { Mechanical circulatory } \\
\text { support }\end{array}$ & $\begin{array}{l}\text { Impact of renal function on eligibility of patients for LVAD implantation, } \\
\text { trajectory of post-LVAD renal function, impact of changes in renal function } \\
\text { (WRF/IRF) on outcomes, and Impella and the kidney }\end{array}$ \\
\hline $\begin{array}{l}\text { Renal replacement therapy } \\
\text { (ADHF) }\end{array}$ & $\begin{array}{l}\text { Choice of RRT (HD, SCUF, or CRRT), customization of UFR, BFR, and DFR in HD, } \\
\text { in-line continuous monitoring of hematocrit and oxygen saturation change, and } \\
\text { urgent-start PD }\end{array}$ \\
\hline Dysnatremia (ADHF) & $\begin{array}{l}\text { Evidence-based use of vasopressin- } 2 \text { receptor antagonists, management of } \\
\text { hypernatremia in fluid overloaded patients, and management of AKI } \\
\text { complicated by hyponatremia }\end{array}$ \\
\hline $\begin{array}{l}\text { Cardiac pressures and } \\
\text { hemodynamics }\end{array}$ & $\begin{array}{l}\text { Familiarity with TTE and TEE, interpretation of various right and left cardiac } \\
\text { pressures and their relation to volume and hemodynamics, kidney in } \\
\text { pulmonary hypertension, and the role of inotropes and their distinctive impact } \\
\text { on various hemodynamic and renal parameters }\end{array}$ \\
\hline \multicolumn{2}{|l|}{ Outpatients } \\
\hline HF co-management & $\begin{array}{l}\text { Use of neprilysin inhibitors, novel agents for chronic hyperkalemia associated } \\
\text { with HF therapy, PD for refractory HF and fluid overload, optimal blood } \\
\text { pressure goals and management, and patients with LVAD as destination } \\
\text { therapy }\end{array}$ \\
\hline ESRD & $\begin{array}{l}\text { Focused diagnosis and classification of HF in the ESRD population, hemodialysis } \\
\text { access placement in severe HF (indications and management), optimization of } \\
\text { PD modalities and regimens for sodium and water removal (e.g., the use of } \\
\text { icodextrin solution), familiarity with the RRT care of patients with } \\
\text { continuous-flow LVADs, and sudden cardiac death }\end{array}$ \\
\hline Heart transplantation & $\begin{array}{l}\text { Antirejection medications and renal function, mTOR inhibitors and proteinuria, } \\
\text { dual heart-kidney transplantation, and CKD care of heart transplant patients }\end{array}$ \\
\hline Pulmonary hypertension & $\begin{array}{l}\text { Familiarity with PHTN groups as related to the kidney, recognizing distinct } \\
\text { effects of PHTN on kidney function, and impact of PHTN therapies on renal } \\
\text { function and outcomes }\end{array}$ \\
\hline Valvular heart disease & $\begin{array}{l}\text { Renal function in various valvular disease (e.g., aortic stenosis and tricuspid } \\
\text { insufficiency) and impact of TAVR and SAVR on the kidney }\end{array}$ \\
\hline Arrhythmias & $\begin{array}{l}\text { Impact on the kidney, renal function and the choice of antiarrhythmic agents, } \\
\text { anticoagulation in patients with renal dysfunction, and ICD and PM placement } \\
\text { in ESRD population }\end{array}$ \\
\hline $\begin{array}{l}\text { End-of-life decisions and } \\
\text { discussions }\end{array}$ & $\begin{array}{l}\text { Patients with cardiorenal syndrome of various etiologies, severity, and } \\
\text { outcomes (e.g., LVAD as destination therapy in irreversible severe kidney } \\
\text { dysfunction or ESRD) }\end{array}$ \\
\hline
\end{tabular}

VC, inferior vena cava; SCUF, slow continuous ultrafiltration; PD, peritoneal dialysis; DOSE, diuretic optimization strategies evaluation; CARRESS-HF, Cardiorenal Rescue Study in Acute Decompensated Heart Failure; WRF, worsening renal function; IRF, improvement in renal function; LVAD, left-ventricular assist device; ADHF, acute decompensated heart failure; RRT, renal replacement therapy; AKI, acute kidney injury; PHTN, pulmonary hypertension; HF, heart failure; mTOR, mechanistic target of rapamycin; CKD, chronic kidney disease; TAVR, transcatheter aortic valve replacement; SAVR, surgical aortic valve replacement; ESRD, end-stage renal disease; ICD, implantable cardioverter defibrillator; PM, pacemaker; CRRT, continuous renal replacement therapy; UFR, ultrafiltration rate; $\mathrm{BFR}$, blood flow rate; $\mathrm{DFR}$, dialysate flow rate. 
on patient outcomes) can also be considered. Co-management of complex ambulatory patients with advanced HF, heart transplant recipients, and those with destination Left Ventricular Assist Device (LVAD) would be integrated into the outpatient component of this curriculum. Topics related to pulmonary hypertension as well as its therapies and their impact on renal function and outcomes are pertinent to this field and need to be included.

The long-range view is for the nephrologist to develop and utilize competence in electrocardiography, echocardiography, vascular ultrasound, and also physiologic studies such as body composition and impedance. While the classically referred studies would still be best performed by experienced specialists in the respective fields, advanced cardiorenal-trained nephrologists would gain competence in performing and interpreting point-of-care ultrasound studies. They can help generate real-time data relevant to the care of the patients in the acute setting. Conversely, the cardiologist would develop and utilize competence in the prescription and management of renal replacement therapies, the care of renal transplant recipients, and the cardiovascular medical and interventional treatment of patients with endstage renal disease.

\section{The Role of Medical Society Support}

The Cardiorenal Society of America (CRSA), the American Society of Nephrology, the National Kidney Foundation, the American College of Cardiology, the American Heart Association, the International Society of Nephrology, and the European Society of Cardiology as well as many other interested medical organizations can all play an important role in the growth and development of cardiorenal medicine. The CRSA has held several "Cardiorenal Universities" to test the interest in and feasibility of cardiologists and nephrologists working together and training with one another to gain skills in electrocardiography, echocardiography, vascular ultrasound, implantable device interrogation, and the use of physiologic studies. Enthusiasm has been high and feedback very positive, particularly among young individuals. These early experiences suggest that we are on the right track with this innovative proposal for a curriculum in cardiorenal medicine.

\section{Conclusion}

The field of cardiorenal medicine is vast, rapidly expanding, and complex. Conventional training programs provide nephrology fellows with the core knowledge necessary for providing general care for patients with cardiovascular diseases. However, there is a need for a focused training of interested physicians to master the specialized aspects of these exceedingly common clinical scenarios, and optimize the care of such patients. A cardionephrologyfocused training will add value to nephrology subspecialties, potentially enhancing its attraction a significant subset of trainees [8].

A nephrologist with advanced cardiorenal training is a valuable asset to any academic institution that seeks to develop interdisciplinary groups for the delivery of comprehensive care to complex patients, in addition to providing focused education to colleagues and trainees at various levels. The added skills of such nephrologists can also help in non-academic settings, with the potential for the enhancement of care metrics, the improvement of professional networking, and serving as a referral point of local or regional significance. Having physicians trained in dedicated cardionephrology tracks will also help improve the design of future cardiorenal medicine studies and enhance the recruitment of patients, a major barrier encountered in this field. 
The creation of an international multidisciplinary group to formulate a comprehensive curriculum for such an educational program is the first step. A variety of practical aspects such as the methods of implementation, the identification of the skills required, and the development of educational assessment tools can be discussed. While this effort will lay the foundation for a specific educational activity (e.g., a specialty track within a nephrology training program), it could also pave the way for the development of an advanced fellowship program in the future.

\section{Statement of Ethics}

The authors have no ethical conflicts to disclose.

\section{Disclosure Statement}

The authors have no potential conflict of interest to declare with respect to this paper.

\section{Funding Sources}

No specific financial support was obtained for preparation of this article.

\section{References}

1 Heidenreich PA, Albert NM, Allen LA, et al; American Heart Association Advocacy Coordinating Committee; Council on Arteriosclerosis, Thrombosis and Vascular Biology; Council on Cardiovascular Radiology and Intervention; Council on Clinical Cardiology; Council on Epidemiology and Prevention; Stroke Council: Forecasting the impact of heart failure in the United States: a policy statement from the American Heart Association. Circ Heart Fail 2013;6:606-619.

2 McCullough PA, Philbin EF, Spertus JA, et al: Confirmation of a heart failure epidemic: findings from the Resource Utilization among Congestive Heart Failure (REACH) study. J Am Coll Cardiol 2002;39:60-69.

3 Ronco C, Haapio M, House AA, et al: Cardiorenal syndrome. J Am Coll Cardiol 2008;52:1527-1539.

4 Kazory A, Elkayam U: Cardiorenal interactions in acute decompensated heart failure: contemporary concepts facing emerging controversies. J Card Fail 2014;20:1004-1011.

5 Ronco C, McCullough P, Anker SD, et al: Cardio-renal syndromes: report from the consensus conference of the Acute Dialysis Quality Initiative (2009). Eur Heart J 2010;31:703-711.

6 Ronco C: The cardiorenal syndrome: basis and common ground for a multidisciplinary patient-oriented therapy. Cardiorenal Med 2011;1:3-4.

7 Ronco C, Ronco F, McCullough PA: A Call to action to develop integrated curricula in cardiorenal medicine. Blood Purif 2017;44:251-259.

8 Rangaswami J, Mathew RO, McCullough PA: Resuscitation for the specialty of nephrology: is cardionephrology the answer? Kidney Int 2018;93:25-26. 